\title{
Terminal Device
}

National Cancer Institute

\section{Source}

National Cancer Institute. Terminal Device. NCI Thesaurus. Code C50216.

An electronic device designed to provide access to a computer. 